\title{
Are Registered Nurses Working in an Acute Care Environment Facing Burnout during the Covid-19 Pandemic?
}

\author{
Abigail Mitchell DHEd, MSN, MBA, RN, CNE* and Kristina N Buran MSN, RN \\ D’Youville College, Buffalo NY, USA
}

*Corresponding author: Abigail Mitchell RN, D’Youville College, Buffalo NY, USA, Email: mitchela@dyc.edu

\section{Research Article \\ Volume 4 Issue 5}

Received Date: October 04, 2021

Published Date: October 21, 2021

DOI: $10.23880 /$ jqhe-16000244

\section{Abstract}

Burnout has been an important and frequently discussed topic in nursing research even prior to the COVID-19 pandemic. Most notably, burnout has previously been identified as one of the major stressors that Registered Nurses have left the acute care field, and now, the COVID-19 pandemic is adding to the already stressful typical workday of an acute care registered nurse. A pilot study was completed on a COVID telemetry unit at a suburban hospital.

Keywords: Nurse Burnout; Nurse Stressors; COVID-19; Intensive Care Units

\section{Overview}

Burnout in nursing has long be a widespread and prevalent topic in the healthcare industry for many years. Nursing is a hard profession, and it is a challenge even on the best days. A Registered Nurse (RN) works in an environment that can change minute by minute. They are required to have expertise with technical skills in addition to the physical strength and mental ability to deal with stress during a crisis. An acute care RN faces even more challenges daily due to nurse to patient ratios, staffing levels, and most importantly, patient acuity. Hospitalized patients typically have a plethora of complex medical histories and diagnoses, need constant monitoring, and have the potential to quickly change from stable to unstable. They must also serve as the liaison between the patient, other healthcare professionals, and the patient's family, who, due to the pandemic concerns, are unable to visit with the patient.

\section{Introduction}

Most of the healthcare workforce consists of Registered Nurses (RNs). There are over 500,000 acute care nurses working in the United States [1]. These nurses are at the bedside to provide care to the patient by demonstrating empathy, compassion, understanding and competence. Patients are very ill. These patients are in serious condition, have a complexity of issues, and need close monitoring. There is a variety of technology to manage. Acute Care Registered Nurses require a variety of skills to deliver the best of care to patients and thrive in fast paced environments. Burnout has been an important topic prior to the pandemic with many unanswered questions. According to a report, 15.6\% of all nurses' report feelings of burnout, with the percentage rising to $41 \%$ of loss of interest in the job [2]. Fast paced environments increased the burnout impact, as it increased the figure by 10 percent. Health care facilities are asking RNs to do more with fewer resources, as currently being seen with the availability of personal protective equipment. Burnout is the state of mental, physical, and emotional exhaustion caused by long hours, fast decision making, and the anxiety for caring for sick patients who many have poor outcomes. The top three common signs of burnout include: emotional exhaustion, depersonalization, and lack of personal accomplishment [3]. This impact is due to the constant awareness and vigilance regarding infection control 
procedures, physical strain of protective equipment, and physical isolation.

\section{Literature Review}

A review of current literature was conducted to determine current research on contributing to nurse burnout. When beginning this research, only a limited number of articles were published, as the COVID19 global pandemic was still a new process. We used keywords in singular formation and in combination; these keywords included: COVID, stress, burnout, COVID19 stress in the work environment, nursing, and staff retention. Registered nurses who work in specialized areas; whether it be medical-surgical, oncology, pediatric, and COVID patients experience stress at high levels due to various demands working with these particular patients. Often in specialized nursing units the complexity of care can create demanding and intense work situations, creating higher burnout rates and stress for those nurses. Depersonalization, which is defined as detachment from others, negative attitudes towards patients or work and lack of empathy, is a common theme among nurses who feel their long hours, demanding roles, lack of breaks and tense clinical situations [4].

Since nurse burnout is a large and evident problem in the profession of nursing, organizations need to have preventative strategies to help reduce burnout. According to a study by Motaghedi, et al. [5] nurses are affected by psychological stress daily. Some causes of stress include: the number of patients, patient needs, regulations and rules that must be followed and the feeling of failure. If this stress is felt daily it can lead to many negative effects on both the nurse experiencing the stress and they may lose their ability to properly care for patients, which can lead to loss of job satisfaction. Acute emotional exhaustion, which often leads to depersonalization of nursing care, is a common occurrence when a nurse is experiencing nurse burnout [6]. Constantly feeling this stress will lead to nurse resilience decreasing, poor job satisfaction and eventually burnout. According to Copanitsanou, et al. [7] a safe work environment is established by having a balanced schedule, professional support, involved management, nurse advancement opportunities, strong skill set and sufficient time to meet all patient needs. According to the review findings by Copanitsanou, et al. [7] nurse burnout is directly affected by the work environment; a positive work environment leads to improved outcomes. Many factors in the environment nurses work play a role in job satisfaction and affect job retention rates. This often changed due to COVID. It was found that most of the previously conducted research centralized around the same conclusions:

- Nurses are experiencing levels of increased stress and worry with COVID19. Contributing factors include staffing levels, complexity of patients diagnosed with COVID19, limited supplies of Personal Protective Equipment (PPE) and ventilators and even Intravenous (IV) pumps, having limited resources available to effectively treat a COVID19 patient [8].

- Many nurses reported having increased anxiety of contracting the virus themselves, or spreading it to family, friends and neighbors.

- New information on the COVID19 virus was frequently changing and new protocols were subsequently implemented.

- Many factors play a role in causing nurse burnout some more influential than others. A very influential factor in nurse burnout is the environment in which a nurse works.

\section{Methodology}

After obtaining the proper Institutional Review Board (IRB) approvals, forty nurses on an identified COVID telemetry unit at a suburban hospital located in New York were surveyed on their thoughts of COVID19 and burnout in nursing. This survey was conducted with an online survey tool using a questionnaire model for 10 questions. This tool sought the nurses' basic demographics and their perception of burnout. The survey size consisted of 23 respondents and consisted of both day shift and night shift registered nurses. Respondents could omit answers, should they wish. The tool was entered an online survey tool for data collection. The researcher emailed a cover letter along with the survey link to all the nurses in the unit. After the data was collected, the nurse's responses were downloaded from the online survey tool and uploaded into statistical software.

\section{Limitations}

- Pilot study, small sample size

- Specific unit

\section{Results}

Over $50 \%$ were under 30 years of age (Figure 1) and $75 \%$ of respondents reported less than 8 years' experience as a Registered Nurse (Figure 2). The responders were relatively new working on the unit, with 14 working less than three years, and of that number, five had only worked for less than one year at the time of survey. Only four responders had worked on the unit for over 12 years. We then asked the survey respondents to comment on a set of questions, thinking prior to the COVID19 outbreak, and during the COVID19 outbreak. The results were overwhelming in terms of numerical data. When posed the question, "Prior to COVID19, do you have feelings of stress, anxiety or sadness prior to your shift on a daily or mostly daily basis", 18 of those surveyed responded 
"No". When asked, "During COVID19, do you have feelings of stress, anxiety or sadness prior to your shift on a daily or mostly daily basis", 19 responded yes.

Many of the Registered Nurses surveyed felt as if they made a difference in their patients' life prior to COVID19 outbreak; in contrast, however, over half of the RNs surveyed did not feel this way during COVID19. Similarly, as most respondents answered "Yes" prior to COVID19 with the question of feeling safe in their working environment, only four RNs stated that yes, they felt safe in their working environment during the peak of the pandemic. We also surveyed to see if these nurses surveyed left their shifts feeling like they completed all their work prior to the COVID19 outbreak. Twenty RNs reported that they felt like they had left their patients in a "safe" environment.

\section{Ages of Respondents}

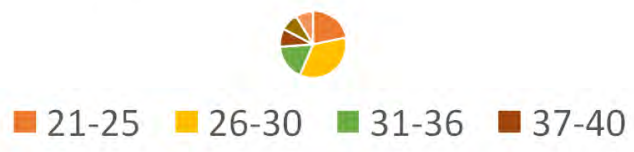

Figure 1: Ages of Respondents.

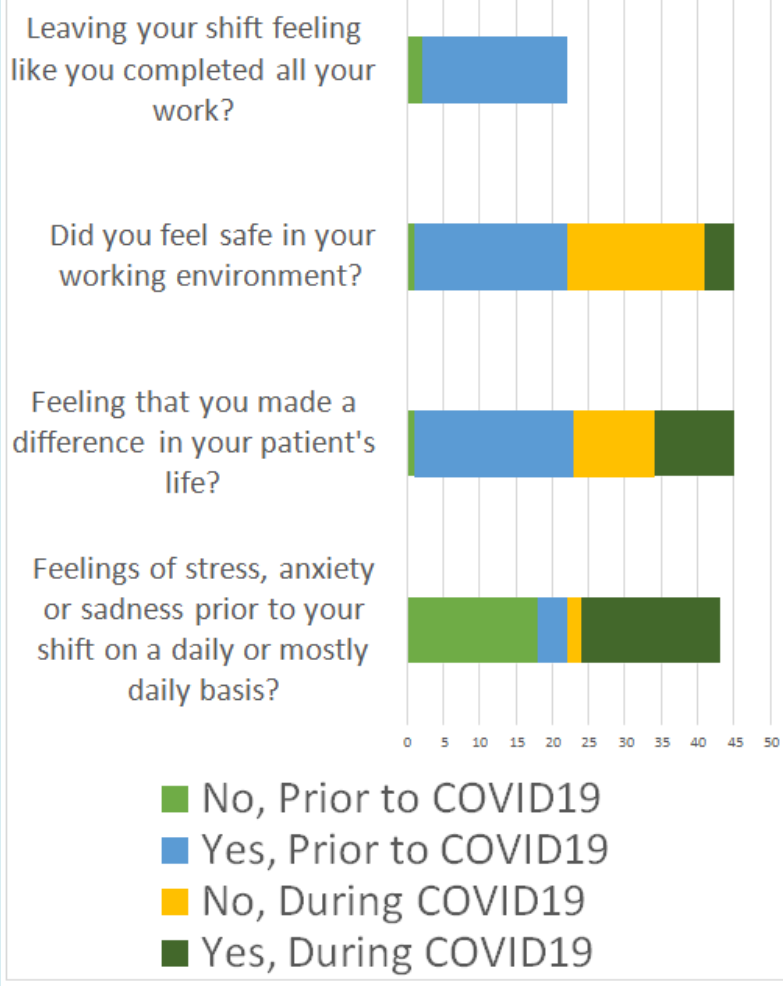

Figure 2: Difference in generations.

\section{Discussion}

The data showed a difference in generations and the discussion of onboarding and orientation should be reviewed. Direct supervision and mentorship for those novice nurses might be helpful in retention. Millennials and Generation $\mathrm{Z}$ nurses want to work in an environment with instant gratification, recognition, work life balance, and in a safe environment [9]. The study demonstrated that many nurses did not believe that they made a difference in their patients' life, and that should be explored. Is it due to staffing? Is it due to stress level of the nurse? Is it due to required personal protective equipment and rooming? Or is it just burnout? Nurse leaders might want to consider looking into the new concept of leadership, appreciative leadership. Appreciative leadership is a positive mental model and framework for leadership practices that are an expression of the response to complexity [10]. All health care professional's role is appreciative in that it is consciously related to the unit. The leader's role includes being a facilitator and each nurse should be aware that they are appreciated for their own contribution to patient outcomes. The leader ensures that all the pieces come together to achieve effectiveness [10].

\section{Conclusion}

More research is needed on this very important topic. The challenges faced by nurses daily are becoming increasingly more stressful. Prior to the COVID19 pandemic, most nurses surveyed reporting not feeling stressed, anxious or sadness on a daily or mostly daily basis; however, during the COVID19 pandemic, the number of nurses with those feelings more than tripled. Most nurses are not feeling safe in their working environment during COVID19. Further studies should include research on what specific stressors that nurses may experience during their shifts, generational gaps, and why they feel unsafe in their work environments. Leaders need to be supportive and demonstrate appreciation on their units. We would like to thank all of the nurses that participated in this study.

Thank you for those Registered Nurses who participated in this project.

\section{References}

1. (2020) American Association of Critical-Care Nurses. Healthy work environments.

2. Copanitsanou P, Fotos N, Brokalaki H (2017) Effects of work environment of patient and nurse outcomes. British Journal of Nursing 26(3): 172-176.

3. Dore C, Duffett Leger L, McKenna M, Breau M (2017) Burnout and empowerment among hemodialysis nurses 
and the current burnout intervention trends: A literature review. Canadian Association of Nephrology Nurses and Technologists 27: 16-31.

4. Foster S (2020b) Burnout: a continuing problem. British Journal of Nursing 29(12): 721.

5. Gordon A, Lyons C, Rao S, Finoli L (2020) Health care workers' challenges in the care of a COVID-19 patient. Critical Care Nursing Quarterly 43(4): 400-406.

6. Kotpa M, Jurkiewicz B, Broda K (2017) Analysis of Factors That Influence the Prevalence of Professional Burnout among Oncology Nurses. Polish Nursing 66: 594- 599.

7. Moses Steele S (2021) Predicting medical-surgical nurses' work satisfaction and intent to stay. Nursing Management 52(9): 17-25.

8. Motaghedi H, Donyavi, Mirzaian B (2016) Effectiveness of mindfulness based cognitive therapy on the distress tolerance of nurses and job burnout. Journal of Nursing and Midwifery Sciences 3(4): 3-12.

9. O Grady Porter T, Malloch K (2021) The emerging principles and practices of appreciative leadership. Nursing Management 52(9): 16-22.

10. Ross J (2020) The exacerbation of burnout during COVID-19: a major concern for nurse safety. Journal of Perianesthesia Nursing 35(4): 439-440. 